\title{
Microwave-assisted synthesis and mechanistic study of multicolor emissive Au nanoclusters using thiol-containing biomolecules
}

\author{
Yong Yu' ${ }^{1}$, Preston Yi Jie Ching ${ }^{1,2}$, Yen Nee Tan ${ }^{1,3 *}$ \\ ${ }^{1}$ Institute of Materials Research and Engineering, $A^{*}$ STAR (Agency for Science, Technology and Research), \\ ${ }^{2}$ Fusion polis Way, 138634 Singapore. \\ ${ }^{2}$ National Junior College, 37 Hillcrest Road, 288913 Singapore. \\ ${ }^{3}$ Department of Chemistry, Faculty of Science, National University of Singapore, 3 Science Drive 3, 117543 Singapore. \\ *Corresponding author
}

DOI: $10.5185 /$ amlett.2018.2081

www.vbripress.com/aml

\begin{abstract}
Developing effective synthesis method for few-atom gold nanoclusters (AuNCs) with tunable emissions is vitally important to gain better understanding of their formation mechanism and provide more design flexibilities to suite for various applications such as multiplex cellular imaging and/or light-emitting diodes. This paper reports a fast method ( $<30$ minutes) of preparing multicolour (red, orange, near infrared) emissive AuNCs via a microwave-assisted biotemplating synthesis approach. A series of analytical tools such as UV-vis and photoluminescence spectroscopies, transmission electron microscopy (TEM) and polyacrylamide gel electrophoresis (PAGE) have been utilized to characterize the resultant AuNCs and unravel the formation mechanism that lead to their multicolour emissive properties. It was found that the surface charge of the thiol-containing peptide reagent and the peptide-to-Au ratio are crucial factors to control the size and emission colour of the resultant AuNCs. By bringing the solution $\mathrm{pH}$ to near the isoelectric point of peptide ligand $(\sim 3)$, the red $\left(\lambda_{\mathrm{em}}=725 \mathrm{~nm}\right)$ emissive AuNC which is initially stable at alkaline conditions (pH12) tends to aggregate due to deficiency of surface charge, thus forming a larger and orange $\left(\lambda_{\mathrm{em}}=640 \mathrm{~nm}\right)$ emissive AuNC. By further applying an even lower peptide-to-Au ratio to fine-tune the protection power of thiolate ligands, a nearinfrared $\left(\lambda_{\mathrm{em}}=846 \mathrm{~nm}\right)$ emissive AuNC has been synthesized. Compared to other microwave-assisted synthesis of AuNCs, current study is featured by its simplicity, rapidity, and versatility to tune the emission wavelength of resultant AuNCs in a much broader range (up to $206 \mathrm{~nm}$ ). Copyright (C) 2018 VBRI Press.
\end{abstract}

Keywords: Gold nanoclusters, tunable emission, microwave-assisted synthesis, biotemplating.

\section{Introduction}

Gold nanoclusters (AuNCs) with a few to a hundred $\mathrm{Au}$ atoms have shown great promise in biological sensing [1-4] and imaging [5-9] due to their ultra-small size $(<2 \mathrm{~nm})$, bright photoluminescence, good photo stability, and low toxicity. These important applications further stimulated the interest of developing effective synthesis methods for fluorescent AuNCs [10-13]. For instance, a biotemplating synthesis approach which utilizes biomolecules such as peptides and proteins to direct the synthesis of fluorescent AuNCs is of particular interest thanks to its inherent biocompatibility and easy functionalization for biological applications [14-15]. In a typical biotemplating method, $\mathrm{Au}$ ions are first sequestered by the functional groups present in the protein or peptide, followed by reduction enabled by the biomolecule itself without the use of an exogenous reducing agent. However, such synthesis process usually takes a relatively long reaction time (hours to days), which restricts the fast acquiring and implementation of fluorescent AuNCs for the intended applications.

More recently, microwave irradiation has been introduced in the biotemplating method for more effective synthesis of fluorescent AuNCs. In a microwave-assisted synthesis, microwave energy is directly coupled with the polar molecules (e.g., water and reagents present in the reaction mixture) to produce efficient internal heating. As such, ultrahigh temperature can be achieved at ambient conditions in a few seconds which greatly accelerates the reaction kinetics to complete the synthesis within a short period of time (from minutes to an hour). Although there are a few studies of combining microwave-assisted method with biomolecules as templates for the synthesis of AuNCs, most of them produce only the AuNCs with single emission color which limit the understanding of their formation mechanism and design flexibilities for specific applications. For example, red emissive AuNCs were synthesized by using lipoic acid [16] or BSA/HSA [17-18] as template. Yellow emissive NCs were 
reported by alloying Ag in BSA-templated AuNCs [19]. Recently, Zhang et al monitored the emission wavelength of a series of AuNCs collected during the course of a microwave-assisted synthesis and observed that the emission of AuNCs could be varied from red $(\sim 670 \mathrm{~nm})$ to orange $(\sim 605 \mathrm{~nm})$ as the irradiation time increased from 10 minutes to 3 hours [20]. However, the quantum yield of those intermediate AuNCs was much lower (e.g., QY $0.42 \%$ for AuNC collected at 10 minutes) than that of the final product (i.e., AuNC collected at $3 \mathrm{~h}$; QY 2.4\%). In addition, the tunable range in emission wavelength was only $65 \mathrm{~nm}$.

Herein, we report a rapid microwave-assisted synthesis of multicolor emissive AuNCs ( $30 \mathrm{~min})$ with a broad range of tunable emission wavelength (up to $206 \mathrm{~nm}$ ) using simple thiol-containing biomolecules, e.g., tripeptide glutathione (GSH) as the template. The control of emission color is achieved by varying the $\mathrm{pH}$ of reaction solution to regulate the overall surface charge of the peptide reagent and the peptide-to-metal ratio. By lowering the solution $\mathrm{pH}$ to near the isoelectric point of the GSH peptide, the red emissive AuNC $\left(\lambda_{\mathrm{em}}=725 \mathrm{~nm}\right)$ initially stable at a higher solution $\mathrm{pH}$ is transformed to orange emissive one $\left(\lambda_{\mathrm{em}}=640 \mathrm{~nm}\right)$. By decreasing the peptide-to-metal ratio further, the emission color of AuNC can be tuned to the near infrared (NIR) region $\left(\lambda_{\mathrm{em}}=846 \mathrm{~nm}\right)$, a biological window where adverse effect of tissue auto fluorescence can be greatly minimized for bio imaging application. The size and optical properties of AuNCs corresponding to different reaction conditions are examined closely to reveal mechanistic insights for the change of emission color. The effect of microwave cleavage of peptide ligand was also studied by replacing GSH with its thiol containing subunits. Present below are the details of this study.

\section{Experimental}

\section{Materials}

Gold (III) chloride solution $\left(\mathrm{HAuCl}_{4}, 30 \%\right.$ in dilute $\mathrm{HCl}$ ), glutathione reduced (GSH, 97\%), and L-cysteine (Cys, 97\%) were purchased from Sigma-Aldrich. Customized peptides Cys-Gly and $\gamma$-Glu-Cys were supplied by Sigma-Aldrich. All reagents were used as received and without further purification. All glassware was washed with Aqua Regia $\left(\mathrm{HCl}: \mathrm{HNO}_{3}\right.$ volume ratio $=3: 1$ ) and rinsed with ethanol and ultrapure water. (Caution: Aqua Regia is a very corrosive oxidizing agent, which should be handled with great care.) Ultrapure water with a specific resistance of $18.2 \mathrm{M} \Omega$ was used throughout the experiment.

\section{Material synthesis}

Tripeptide $\gamma$-Glu-Cys-Gly or GSH was selected as a model thiol-containing biomolecule for the synthesis of multicolor emissive AuNCs. Aqueous solutions of $\mathrm{HAuCl}_{4}(25 \mu \mathrm{L}, 20 \mathrm{mM})$ and $\mathrm{GSH}(25 / 37.5 / 50 \mu \mathrm{L}, 20$ $\mathrm{mM})$ were mixed with a GSH-to-Au ratio $\left(R_{[\mathrm{GSH}] /[\mathrm{Au}]}\right)$ of
$1: 1,1.5: 1$ or $2: 1$ in a $1.5 \mathrm{~mL}$ glass vial under vigorous, constant stirring. The reaction mixture was topped up to $1 \mathrm{~mL}$ with ultrapure water. After that, the solution $\mathrm{pH}$ was adjusted with $\mathrm{NaOH}(1 \mathrm{M})$ to 3,9 and 12 prior to microwave irradiation. The synthesis was carried out in a domestic Cornell DMO-4099 Microwave Oven under intermittent irradiation for $4 \times 1$ minute at $385 \mathrm{~W}$. The synthesis conditions and appearance of resulting AuNCs are summarized in Scheme 1. Specifically, the red emissive AuNC (r-AuNC) was formed with $R_{[G S H] /[A u]}=1.5: 1$ at $\mathrm{pH} 12$ and was collected right after microwave heating. The orange emissive AuNC (o-AuNC) was formed with a same $R_{[G S H] /[A u]}$ as the red emissive one but at a lowered solution pH (i.e., 3), and was collected 30 minutes after irradiation. The nearinfrared emissive AuNC (NIR-AuNC) was synthesized with a lower $R_{[G S H] /[A u]}(1: 1)$ at $\mathrm{pH} 3$ and was also collected immediately after microwave irradiation All samples are centrifuged at 8,000 rpm for 10 minutes to remove any large particles.

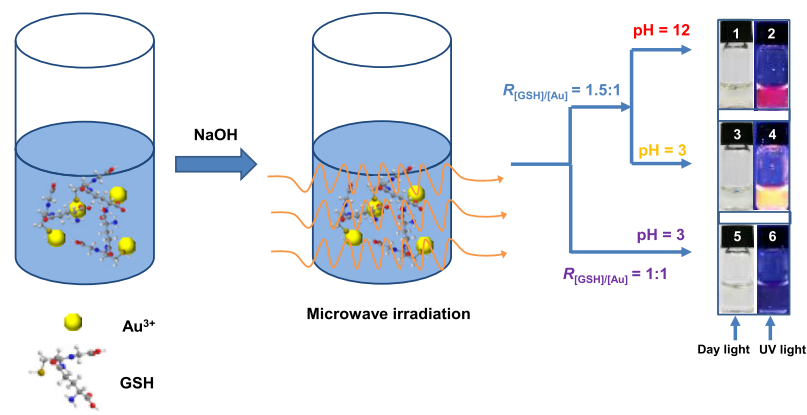

Scheme 1. Schematic diagram of the microwave-assisted synthesis of $(\mathbf{1}, \mathbf{2})$ red, $(\mathbf{3}, \mathbf{4})$ orange, and $(\mathbf{5}, \mathbf{6})$ near-infrared emissive AuNCs.

\section{Characterizations}

Unless otherwise specified, UV-vis absorption and photoemission spectra of gold nanoclusters were obtained on a Tecan microplate reader. The photoemission spectrum of near infrared emitting AuNC was measured on a Horiba Jobin Yvon Fluorolog-3 spectrofluorometric. The quantum yield (QY) of AuNCs was measured using a $370 \mathrm{~nm}$ Xenon laser as light source and 4-(Dicyanomethylene)-2methyl-6-(4-dimethylaminostyryl)-4H-pyran (DCM) as reference. The QY of AuNCs was calculated based on a comparative method and using Equation (1) below.

$$
Q_{S}=Q_{R} \times \frac{k_{S}}{k_{R}} \times \frac{n_{S}^{2}}{n_{R}^{2}}
$$

where $Q_{R}$ is the known quantum yield of the reference compound, $\mathrm{k}$ is slope of the lines obtained from the plot of the integrated fluorescence intensity $\left(\lambda_{\mathrm{ex}}=370 \mathrm{~nm}\right)$ vs. absorbance (at $370 \mathrm{~nm}$ ), $n$ is the refractive index of solvent, and the subscripts $\mathrm{S}$ and $\mathrm{R}$ refer to sample and reference, respectively. Transmission electron microscopy (TEM) images were taken on a JEOL JEM2010 microscope operating at $200 \mathrm{kV}$. Native 
polyacrylamide gel electrophoresis (PAGE) was carried out on a Bio-Rad Mini-PROTEAN Tetra Cell system. Stacking and resolving gels were prepared from 4 and 30 wt $\%$ acrylamide monomers, respectively. The electrophoresis was allowed to run for $\sim 2.5 \mathrm{~h}$ at a constant voltage of $150 \mathrm{~V}$.
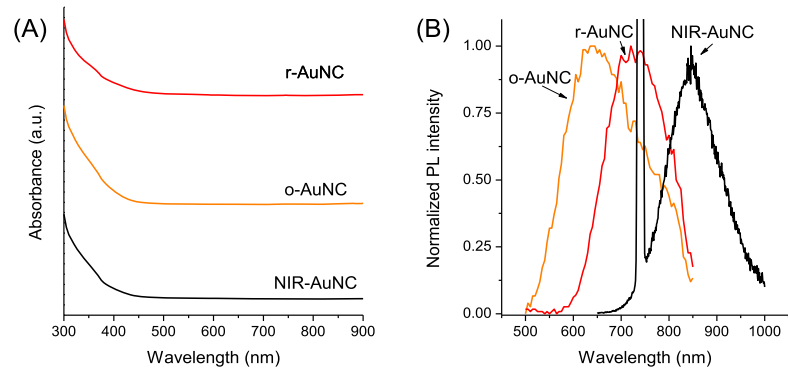

Fig. 1. (A) UV-vis and (B) photoemission spectra of red, orange, and near infrared emissive Au NCs synthesized by a microwave-assisted method using GSH tripeptide as template.

\section{Results and discussion}

Fig. 1A shows the UV-vis absorption spectra of the red, orange, and near-infrared emissive AuNCs synthesized by the microwave-assisted method using GSH as the model bimolecular template in this study. It is well known that large $\mathrm{Au}$ nanoparticles $(>2 \mathrm{~nm})$ show a distinct peak at $\sim 520 \mathrm{~nm}$ on the photo absorption spectra due to the surface plasma resonance (SPR) of their conduction electrons [21]. However, no obvious peaks were observed on the UV-vis absorption spectra of any AuNCs in this study, which suggest the AuNCs are really small in size such that the Au atoms in these AuNCs are so few to support SPR. The molecular-like emissions of these AuNCs were further characterized by the photoluminescence measurement. The emission peaks were observed at an emission maximum $\left(\lambda_{\mathrm{em}}\right)$ of $725 \mathrm{~nm}, 640 \mathrm{~nm}$, and $846 \mathrm{~nm}$ for the red, orange, and near-infrared emissive AuNCs, respectively (Fig.1B). These emission peaks were not observed in the control experiments with only GSH, or gold chloride included in the reaction solution, which confirmed that the emissions were exclusively from the as-formed AuNCs in the respective reaction mixture. The quantum yield of the red, orange, and near-infrared AuNCs was calculated to be $2.56 \%, 3.12 \%$, and $2.69 \%$, respectively.

Fig. 2 show the TEM images and size distribution histograms of the as-synthesized red, orange, and nearinfrared emissive AuNCs. As expected, all the three types of AuNCs are very small in size and most particles are within the sub-2 nm size range. Further analysis of the size distribution gave an average size of $0.53 \pm 0.04 \mathrm{~nm}, 0.84 \pm 0.06 \mathrm{~nm}$, and $1.03 \pm 0.03 \mathrm{~nm}$ for the red, orange, and near-infrared emissive AuNCs, respectively. Note that high resolution TEM images are not shown because the AuNCs tended to fuse into larger particles when they were shone with an electron beam with higher energies. Surprisingly, the sizes of the red, orange, and near-infrared AuNCs does not follow the trend suggested by the previously reported Jellium model [22], in which the emission energy $(E)$ of AuNCs is proposed to be inversely proportional to their sizes $(N)$ via the relation $E=E_{\mathrm{F}} / N^{1 / 3}$, where $E_{\mathrm{F}}$ is the Fermi energy of the bulk metal (i.e., Au).

The unique size-emission correlation of these multicolor emissive AuNCs has motivated us to further examine the synthesis conditions and their formation mechanism. GSH is a naturally occurring tripeptide $(\gamma$ Glu-Cys-Gly) having two $-\mathrm{COOH}$, one $-\mathrm{NH}_{2}$, and one $-\mathrm{SH}$ group. The thiol group is well known for its reducing capability to reduce $\mathrm{Au}(\mathrm{III})$ to $\mathrm{Au}(\mathrm{I})$ [23]. As a result, stable $\mathrm{Au}(\mathrm{I})-\mathrm{SG}$ complexes are formed at ambient conditions (SG denotes the deprotonated thiolated anion of GSH). As microwave irradiation is extremely efficient, super heating can be built up in as short as seconds, these $\mathrm{Au}(\mathrm{I})-\mathrm{SG}$ complexes could absorb the energy from the microwave irradiation, leading to the activation of the functional groups on the thiolates to donate electrons to $\mathrm{Au}(\mathrm{I})$ in forming $\mathrm{Au}(0)$. Thus, ultra-small AuNCs could be formed in minutes by the aggregation of these in-situ formed $\mathrm{Au}$ atoms.
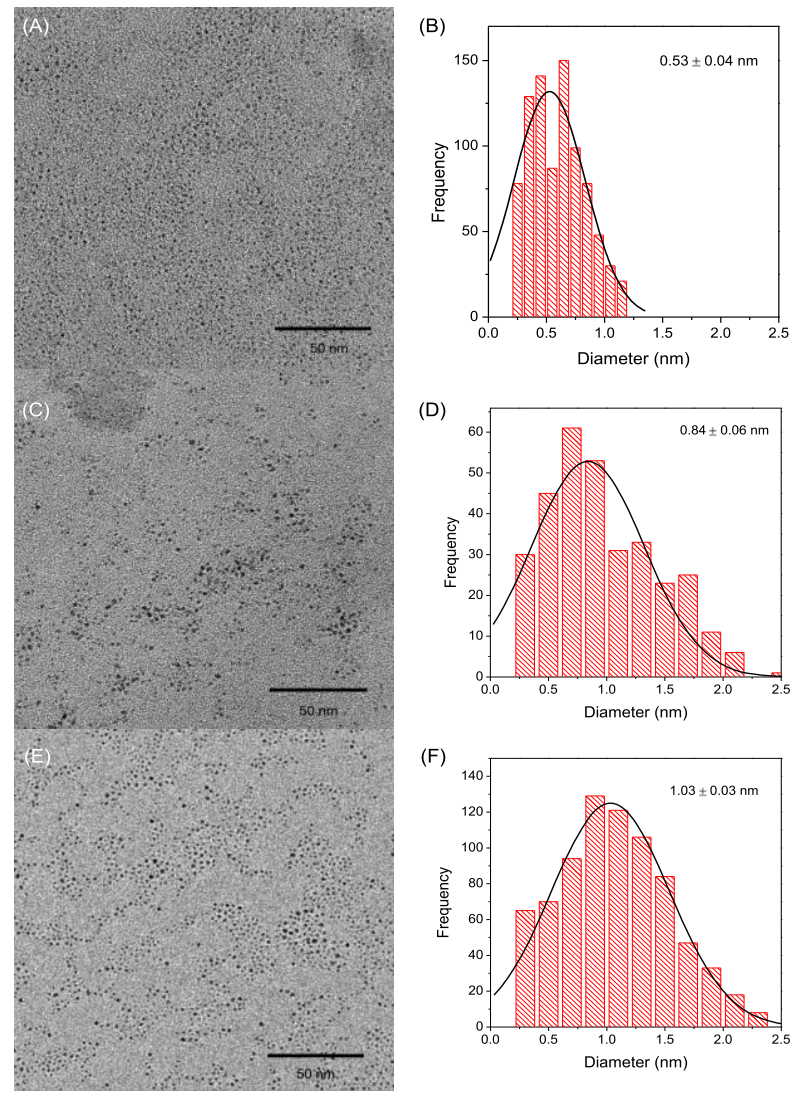

Fig. 2. (A, C, E) TEM images and (B, D, F) size histograms of (A, B) red, $(C, D)$ orange, and $(E, F)$ near infrared emissive Au NCs.

As GSH has three types of functional groups with different $p K_{a}$ values (Fig. 3A), the impact of the solution $\mathrm{pH}$ to the formation of different emissive AuNCs was further examined. Base on the molecular structure, GSH has an isoelectronic point of 2.85. When the solution $\mathrm{pH}$ was 3 , only the $-\mathrm{COOH}$ group in Glu was deprotonated and the net charge of GSH was 0 . As 
a result, the GSH-protected AuNCs tend to aggregate into larger ones due to their poor dispersity arising from their charge neutral surface.

Interestingly, a red $\rightarrow$ orange emission change $(725 \mathrm{~nm} \rightarrow 640 \mathrm{~nm})$ of the AuNC prepared at $R_{[\mathrm{GSH}] / \mathrm{Au}]}=1.5: 1$ and $\mathrm{pH} 3$ was observed in about 30 minutes (Fig. 3B). Note that blue-shift in emission is typical for AuNCs with aggregation induced emission (AIE) behaviors. Clustering of the primary AuNCs was also observed in the TEM image of the orange emissive AuNC (Fig. 2B). Polyacrylamide gel electrophoresis (PAGE) analysis further confirmed the AIE properties of the orange emissive AuNC. A series of AuNCs with different sizes (i.e., $\mathrm{Au}_{15}(\mathrm{SG})_{13}, \mathrm{Au}_{18}(\mathrm{SG})_{14}$, $\mathrm{Au}_{22}(\mathrm{SG})_{16-17}, \mathrm{Au}_{25}(\mathrm{SG})_{18}, \mathrm{Au}_{29}(\mathrm{SG})_{20}, \mathrm{Au}_{33}(\mathrm{SG})_{22}$ and $\left.\mathrm{Au}_{39}(\mathrm{SG})_{24}\right)$ by using a previously reported protocol [24] were used as a reference to estimate the size of the orange emissive AuNCs. As shown in Fig. 3C, the orange emissive AuNC (lane II and IV) contains two prominent bands with a migration distance smaller than $\mathrm{Au}_{29}(\mathrm{SG})_{20}$ but larger than $\mathrm{Au}_{39}(\mathrm{SG})_{24}$, suggesting that these two species contain more than 29 but less than $39 \mathrm{Au}$ atoms.
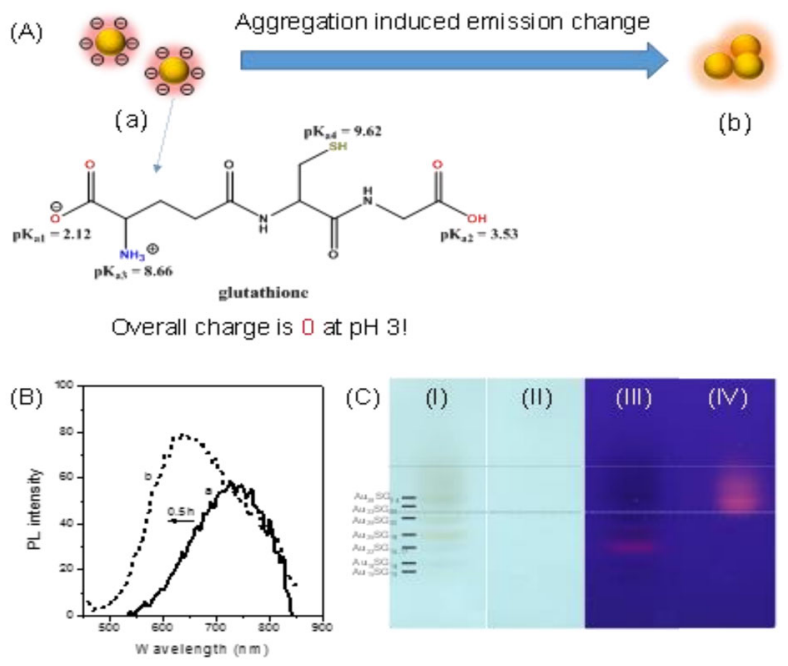

Fig. 3. (A) Scheme showing the emission change of AuNCs effected by charge induced aggregation (Reaction conditions: $R_{[G S H] /[A u]}=$ 1.5:1, pH 3; a-immediately synthesized, b- after aging for 30 minutes). (B) Corresponding UV-vis spectra of the AuNCs in (A). (C) PAGE gels of (lane I, III) mix-sized $\mathrm{Au}_{\mathrm{n}}(\mathrm{SG})_{\mathrm{m}}$ prepared according to a published protocol, and (lane II and IV) the orange emissive Au NCs under (I, II) visible and (III, IV) UV light.

A smeared band with an even greater migration distance than $\mathrm{Au}_{39}(\mathrm{SG})_{24}$ was also observed, which suggested that some larger species were also presented in the orange emissive AuNC, but they couldn't be separated by PAGE at the investigated conditions. Nonetheless, all these species showed similar orange emissions regardless of their size difference, which is another characteristic of AIE-type AuNCs [25]. Therefore, it is concluded that the red emissive AuNCs were less stable at the isoelectronic point of GSH, which gradually aggregate and reorganize into orange emissive AuNCs under the acidic conditions $(\mathrm{pH} 3)$.
When the solution $\mathrm{pH}$ was 12 , all of the functional groups of GSH were deprotonated with a net charge of -2 . In this case, the negatively charged red emissive AuNCs is found to be more stable at high $\mathrm{pH}$ conditions (pH 12) with no color change observed during the course of reaction. It is therefore speculated that the surface charge of the AuNCs is critical to determine the emission color of the final product.

When $R_{[\mathrm{GSH}] /[\mathrm{Au}]}$ was further decreased to $1: 1$ while $\mathrm{pH}$ was kept as 3, NIR emissive AuNCs with the largest atomic size were observed. As the amount of GSH is relevant to the polymerization degree of the $\mathrm{Au}(\mathrm{I})$ thiolate complexes, a lower $R_{[\mathrm{GSH}] /[\mathrm{Au}]}$ ratio will render the $\mathrm{Au}(\mathrm{I})$-thiolate complexes to get reduced easier in forming more $\mathrm{Au}(0)$. On the other hand, a lower $R_{[\mathrm{GSH}][\mathrm{Au}]}$ ratio also gives less thiolate motifs available to cap on the in-situ formed $\mathrm{Au}(0)$, and thus a lesser protection to prevent the growth of in-situ formed AuNCs into larger particles. As a result, a higher chance of these $\mathrm{Au}(0)$ to collide with each other in the presence of limited protecting motifs lead to the formation of larger AuNCs that give the NIR emissions.
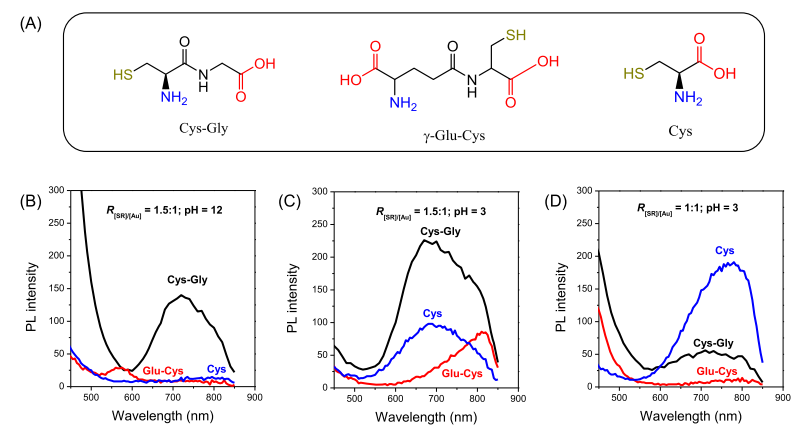

Fig. 4. (A) Molecular structures of Cys-Gly, $\gamma$-Glu-Cys, and Cys. (B-D) PL spectra of AuNCs synthesized with corresponding peptides/amino acids at various different reaction conditions (B: $R_{[S R] /[A u]}=1.5: 1, \mathrm{pH} 12 ; \mathrm{C}: R_{[S R] /[A u]}=1.5: 1, \mathrm{pH} 3 ; \mathrm{D}: R_{[S R] /[A u]}=$ $1: 1, \mathrm{pH} 3)$.

Other than the surface charge and relative strengths of the particle growth and passivation, the possible decomposition of GSH under microwave irradiation may also be accounted for the formation of multicolor emissive AuNCs. Failure et al. discovered that reduced GSH could be decomposed at sterilization temperatures to release amino acids other than forming oxidized form of GSH, i.e., GS-SG [26]. The super high temperature created by microwave irradiation in this work could also lead to the decomposition of GSH via cleavage at different amide bond sites to produce bipeptides or amino acids that might participate in the formation of AuNCs. To investigate the possible impact of these GSH derived peptides or amino acids on the formation of AuNCs, we replaced GSH with three possible thiolcontaining compounds, i.e., Cys-Gly, Glu-Cys and Cys (only thiol-containing compounds were selected due to the essential role of strong $\mathrm{Au}-\mathrm{S}$ interactions in the formation of AuNCs, see their molecular structures in Fig. 4A) in the microwave-assisted synthesis. At the same synthesis conditions for r-AuNC with GSH (i.e., 
$R_{[\mathrm{SR}][\mathrm{Au}]}=1.5: 1, \mathrm{pH} 12$; here $\mathrm{H}-\mathrm{SR}$ denotes the respective thiol-containing compound), only Cys-Gly led to the formation of a red emissive AuNC with an emission maxima at $\sim 720 \mathrm{~nm}$ while the other two did not produce any intensely fluorescent species (Fig. 4B). While at the same conditions for o-AuNC with GSH (i.e., $R_{[\mathrm{SR}][\mathrm{Au}]}=1.5: 1, \mathrm{pH} 3$ ), both Cys-Gly and Cys produced red emissive AuNCs with an emission maxima at $\sim 685 \mathrm{~nm}$, while a NIR emissive AuNC with an emission maxima at $\sim 815 \mathrm{~nm}$ was formed for GluCys under the same reaction conditions (Fig. 4C). Note that a blue-shift in emission maxima was observed for Cys-Gly when reaction $\mathrm{pH}$ was changed from 12 to 3, which is similar to the phenomenon observed for GSH. As Cys-Gly possesses no net charge at $\mathrm{pH} 3$ either, it might also arise from the surface charge mediated aggregation. At the same synthesis conditions for NIRAuNC with GSH (i.e., $R_{[\mathrm{SR}][\mathrm{Au}]}=1: 1, \mathrm{pH} 3$ ), a NIR emissive AuNC with an emission maximum at $780 \mathrm{~nm}$ was also observed for Cys, the wavelength of which is $95 \mathrm{~nm}$ longer than that produced with Cys with a higher peptide-to-metal ratio at the same solution $\mathrm{pH}$ (i.e., $R_{[\mathrm{SR}][\mathrm{Au}]}=1.5: 1$ and $\mathrm{pH} 3$, Fig. 4D). This phenomenon is also corroborated with that observed in the case of $\mathrm{GSH}$, which is due to weaker protection of less thiolate ligands. Only a weakly red emissive AuNC was formed for Cys-Gly $\left(\lambda_{\mathrm{em}}=\sim 720 \mathrm{~nm}\right)$ while no fluorescent species was formed for Glu-Cys. These results suggest the overall surface charge of peptide reagent and the peptide-to-metal ratio still dominate the emission color of final products even cleavage of GSH could occur under microwave irradiation.

\section{Conclusion}

In summary, we presented here the one-pot synthesis of multicolor (red, orange, and NIR) emissive AuNCs with a simple bio-occurring peptide in a microwaveassisted method. It was found that the overall charge of peptide played an important role in controlling the emission color of resultant AuNCs. When the solution $\mathrm{pH}$ was regulated near the isoelectric point and thus there was no net charge for the peptide ligand, the red emissive AuNC which was stable at a higher solution $\mathrm{pH}$ (12) aggregated into larger and orange emissive AuNC. Transmission electron microscopy (TEM), photoluminescence (PL) and polyacrylamide gel electrophoresis (PAGE) studies revealed that the blueshift in emission color was due to the mechanism of aggregation induced emission (AIE). The emission color of AuNCs was further tuned to the near infrared (NIR) region by varying the peptide-to-metal ratio to control the relative strength of protecting thiolate ligands. Investigations of replacing GSH with its thiolcontaining subunits suggest that the overall surface charge of peptide and peptide-to-metal ratio other than the sequence are more critical factors to control the emission color of resultant AuNCs. This study is of interest not only because it can supply AuNCs with tunable emissions in a simple and rapid way, but also because it exemplifies the important role of overall surface charge of peptide reagent in determining the emission color of AuNCs, which can be simply controlled by varying the solution $\mathrm{pH}$.

\section{Acknowledgements}

This work was financially supported by Institute of Materials Research and Engineering, Agency of Science, Technology and Research (A*STAR), under Boinspired Approaches to Biomimetic Materials program (IMRE/00-1P1400).

\section{Author's contributions}

Conceived the plan: YY, YNT; Performed the expeirments: YY, YJC; Data analysis: YY, YNT; Wrote the paper: YY, YNT. Authors have no competing financial interests.

\section{References}

1. Xie, J., Zheng, Y. and Ying, J. Y.; Chem. Commun. 2010, 46, 961.

2. Wang, S., Meng, X., Feng, Y., Sheng, H. and Zhu, M.; RSC Adv. 2014, 4, 9680 .

3. Yu, Y., New, S. Y., Xie, J., Su, X. and Tan, Y. N.; Chem. Commun. 2014, 50, 13805.

4. Chang, H.-C., Chang, Y.-F., Fan, N.-C. and Ho, J.-a. A.; $A C S$ Appl. Mater. Interfaces 2014, 6, 18824.

5. Frangioni, J. V.; Curr. Opin. Chem. Biol. 2003, 7, 626.

6. Sun, C., Yang, H., Yuan, Y., Tian, X., Wang, L., Guo, Y., Xu, L., Lei, J., Gao, N., Anderson, G. J., Liang, X.-J., Chen, C., Zhao, Y. and Nie, G.; J. Am. Chem. Soc. 2011, 133, 8617.

7. Shang, L., Yang, L., Wang, H. and Nienhaus, G. U.; Small 2016, 12,868 .

8. Yu, Y., Zheng, X. T., Yee, B. W. and Tan, Y. N.; Mater. Sci. Eng. C 2017, 77, 1111.

9. Shen, D., Henry, M., Trouillet, V., Comby-Zerbino, C., Bertorelle, F., Sancey, L., Antoine, R., Coll, J.-L., Josserand, V. and Guével, X. L.; APL Mater. 2017, 5, 053404.

10. Yu, Y., Yao, Q., Luo, Z., Yuan, X., Lee, J. Y. and Xie, J.; Nanoscale 2013, 5, 4606.

11. Yuan, Q., Wang, Y., Zhao, L., Liu, R., Gao, F., Gao, L. and Gao, X.; Nanoscale 2016, 8, 12095.

12. Pyo, K., Ly, N. H., Yoon, S. Y., Shen, Y., Choi, S. Y., Lee, S. Y., Joo, S.-W. and Lee, D.; Adv. Healthcare Mater. 2017, 6, 1700203.

13. Ghosh, D., Baksi, A., Mudedla, S. K., Nag, A., Ganayee, M. A., Subramanian, V. and Pradeep, T.; J. Phys. Chem. C 2017, 121, 13335.

14. Xie, J., Zheng, Y. and Ying, J. Y.; J. Am. Chem. Soc. 2009, 131, 888.

15. Yu, Y., Mok, B. Y. L., Loh, X. J. and Tan, Y. N.; $A d v$. Healthcare Mater. 2016, 5, 1844.

16. Shang, L., Yang, L., Stockmar, F., Popescu, R., Trouillet, V., Bruns, M., Gerthsen, D. and Nienhaus, G. U.; Nanoscale 2012, 4, 4155.

17. Yan, L., Cai, Y., Zheng, B., Yuan, H., Guo, Y., Xiao, D. and Choi, M. M. F.; J. Mater. Chem. 2012, 22, 1000.

18. Yue, Y., Liu, T. Y., Li, H. W., Liu, Z. Y. and Wu, Y. Q.; Nanoscale 2012, 4, 2251.

19. Zheng, B., Zheng, J., Yu, T., Sang, A., Du, J., Guo, Y., Xiao, D. and Choi, M. M. F.; Sens. Actuators, B 2015, 221, 386.

20. Zhang, J., Yuan, Y., Liang, G., Arshad, M. N., Albar, H. A., Sobahi, T. R. and Yu, S.-H.; Chem. Commun. 2015, 51, 10539.

21. Tan, Y. N., Lee, K. H. and Su, X.; Anal. Chem. 2011, 83, 4251.

22. Zheng, J., Zhang, C. and Dickson, R. M.; Phys. Rev. Lett. 2004, 93, 077402 .

23. Schaaff, T. G., Knight, G., Shafigullin, M. N., Borkman, R. F. and Whetten, R. L.; J. Phys. Chem. B 1998, 102, 10643.

24. Negishi, Y., Nobusada, K. and Tsukuda, T.; J. Am. Chem. Soc. 2005, 127,5261 .

25. Luo, Z., Yuan, X., Yu, Y., Zhang, Q., Leong, D. T., Lee, J. Y. and Xie, J.; J. Am. Chem. Soc. 2012, 134, 16662.

26. Failure, A., Postaire, E., Prognon, P., Pradier, F. and Pradeau, D.; J. Pharm. Biomed. Anal. 1992, 10, 457. 